\title{
RV Tauri stars and Type II Cepheids in the LMC
}

\author{
Karen R. Pollard \\ Department of Physics and Astronomy, University of Canterbury, \\ Private Bag 4800, Christchurch, New Zealand \\ T. Lloyd Evans \\ South African Astronomical Observatory, PO Box 9, Observatory 7935, \\ South Africa
}

\begin{abstract}
A review of the main results from the MACHO project for RV Tauri stars and Type II Cepheids in the LMC is presented. In light and colour curve behaviour, the RV Tauri stars appear to be a direct extension of the Type II Cepheids to longer periods. A single periodluminosity-colour relationship is seen to describe both the Type II Cepheids and RV Tauri stars in the LMC.

Follow-up low resolution spectroscopy of the LMC RV Tauri stars has been undertaken. The spectra of the longest period RV Tauri star in the MACHO sample show that this star has strong $\mathrm{C}_{2}$ bands with a pronounced phase dependence, their strength being greater at minimum light. This and other spectral features are similar to those observed in proto-planetary nebulae. This LMC star therefore appears to combine the main spectral anomaly of the proto-planetary nebulae with RV Tauri-like photometric variability. This is an intriguing result which indicates a possible link between RV Tauri stars and proto-planetary nebulae, and strengthens the post-AGB interpretation of the RV Tauri stars.
\end{abstract}

\section{Introduction}

RV Tauri stars are luminous, long-period members of the Type II class of Cepheid variables. The shorter-period stars of the $\mathrm{W}$ Vir class are hydrogen- and heliumshell burning stars (Schwarzchild \& Harm 1970) while the RV Tauri stars are believed to be in a more advanced stage of evolution at the end of the asymptotic giant branch (AGB) (Gingold 1974; Jura 1986). The AGB stars consist of a $\mathrm{C}-\mathrm{O}$ degenerate core with a thin overlying helium layer inside a large tenuous convective hydrogen envelope. The star evolves off the AGB after severe mass loss from the envelope. An RV Tauri variable may be a post-AGB star with a necessarily low-mass but extended envelope. The surface composition of such a star would be expected to reflect that of its cool AGB predecessor, but with a far simpler spectrum due to its warmer temperature.

Recent high-resolution abundance studies of galactic RV Tauri stars (Gonzalez, Lambert \& Giridhar 1997a, b) have shown, however, that many field RV Tauri stars have atmospheres that are preferentially depleted of elements of 
high condensation temperature. Intriguingly, further studies to examine these abundances (Gonzalez \& Lambert 1997; Russell 1997) have found evidence that this preferential depletion does not appear to occur in globular cluster RV Tauri variables or high-velocity field stars, indicating that the low initial metallicity of the progenitor star may be an inhibiting factor in this depletion process.

\subsection{RV Tauri stars in the Large Magellanic Cloud}

The discovery of RV Tauri stars in the Large Magellanic Cloud (LMC) was announced recently (Pollard et al. 1997; Alcock et al. 1998). This discovery has finally allowed us to make a definitive assessment of the luminosities of these stars which will help us in our investigation of the other fundamental parameters. The derived absolute magnitude of about -4.5 for these variables at a fundamental period of $\sim 50$ days lends strong support to the proposal that these stars are luminous post-AGB stars evolving to the left in the HR diagram. Further results from this analysis of the MACHO database were that there was a progression in behaviour from the short period Type II Cepheids to the longer period RV Tauri stars: in terms of their light- and colour-curve properties the RV Tauri stars were found to be a direct extension of the Type II Cepheids to longer periods. A period-luminosity-colour relationship of the form

$$
M_{V}=-0.61-2.95 \log P+5.49<(V-R)_{0}>
$$

gave an extremely tight relation for both the Type II Cepheids and RV Tauri stars in the LMC. Further details can be found in Pollard et al. (1997) and Alcock et al. (1998).

This paper presents the first spectroscopic results obtained for an RV Tauri star in the LMC. MACHO*04:55:43.2-67:51:10 has a formal period of 112.45 days and is the longest period variable from the Alcock et al. (1998) sample of 33 Type II Cepheid and RV Tauri stars in the LMC. The light curve is shown in Fig. 1. The solid curve plotted in this figure is the 5th order Fourier fit to the optical $\left(V_{\mathrm{M}}\right)$ photometry from the MACHO collaboration. The light and colour variations in this star are similar to the behaviour typical of galactic RV Tauri variables (Pollard et al. 1996). However, the low-resolution spectra of this object are most unusual and unlike any spectra that have been obtained previously of galactic RV Tauri stars (Pollard et al. 1997 and references therein).

\section{Observations}

Low-resolution optical spectra and infrared JHK photometry were obtained of the LMC RV Tauri variable star MACHO*04:55:43.2-67:51:10 in order to examine the overall spectral characteristics and infrared properties. Three spectra were obtained with the Unit Spectrograph and RPCS detector on the $1.9-\mathrm{m}$ reflector at the South African Astronomical Observatory (SAAO) using a $7 \AA$ resolution over the range $3500-6500 \AA$ approximately. These spectrograms were obtained during 1996 December to 1997 February at widely-separated phases of $0.61,0.87$ and 1.16 (where a phase of 0.0 corresponds to the deep or 'primary' minimum). Infrared $J H K$ observations of MACHO*04:55:43.2-67:51:10 were taken during 1996 December using the Mk III infrared photometer on the SAAO 1.9-m telescope, when the star was close to maximum light (phase 0.69). 


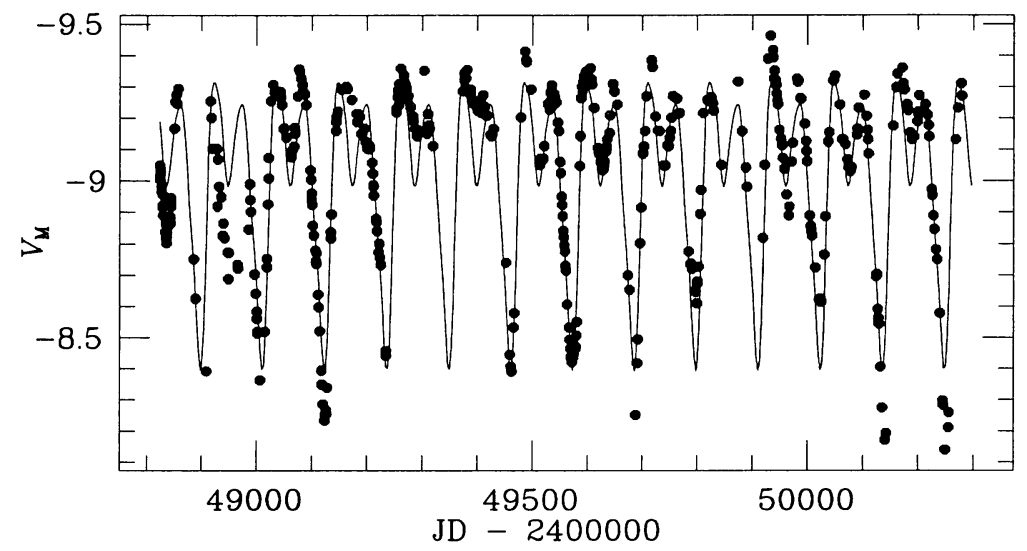

Figure 1. The raw $V_{\mathrm{M}}$ light curve for MACHO*04:55:43.2-67:51:10 plotted together with the 5 th order Fourier fit to the photometric data. This star has a double or 'formal' period of 112.45 days.

\section{Results}

\subsection{Spectral classification}

The three spectra of the LMC RV Tauri star MACHO*04:55:43.2-67:51:10 were compared to a sequence of standard star spectra of supergiants of luminosity class Ib, using the Balmer lines of $\mathrm{H}, \mathrm{Ca}$ II, $\mathrm{CH}$ and $\mathrm{CN}$. The low resolution and $\mathrm{S} / \mathrm{N}$ of these spectra does not permit the use of the weaker metallic lines. The features used do not yield a consistent result, a common situation with Type II Cepheids and RV Tauri stars whose spectra are affected by shock-wave phenomena as well as by the abundance anomalies referred to in the Introduction.

The weak Ca II lines in the December spectrum indicate an early $\mathrm{F}$ type, perhaps F3, and the weak CN bands indicate a type earlier than G0. The weak Balmer lines of $\mathrm{H}$ are not consistent with any type earlier than G2 while the G-band of $\mathrm{CH}$ suggests G0, however. The two subsequent spectra, especially that of 10 February, are a little later in spectral type (that is, apart from the carbon bands). The estimated $\left(V-R_{\mathrm{c}}\right)_{0}$ colour of 0.27 on 10 December gives a spectral class of $\mathrm{F} 9 \mathrm{Ib}$ (Cousins 1981), while the $(B-V)_{s p, 0}$ of 0.74 on the same date is consistent with this result, giving a spectral class of G0 Ib.

Comparison was made with the spectra of other carbon-rich variables. The three low-resolution spectra of the LMC RV Tauri star MACHO*04:55:43.267:51:10 are shown in Fig. 2, together with those of the $\mathrm{R}$ Coronae Borealis (RCB) star V517 Oph (Kilkenny et al. 1992) and the galactic RV Tauri star SX Cen. The principal absorption features are marked. Neither of the galactic stars are a particularly good match for MACHO*04:55:43.2-67:51:10.

No galactic RV Tauri star is known to have $\mathrm{C}_{2}$ bands as strong as those in MACHO*04:55:43.2-67:51:10. Such strong $\mathrm{C}_{2}$ bands normally imply very strong $C N$ bands at 4215 and $3883 \AA$. Unusually, the presence of $C N$ in the spectrum is only marginal. In addition, $\mathrm{CH}$ is represented in this star only by a weak $\mathrm{G}$ band. The Balmer lines of $\mathrm{H}$ are very weak in the two spectra with 


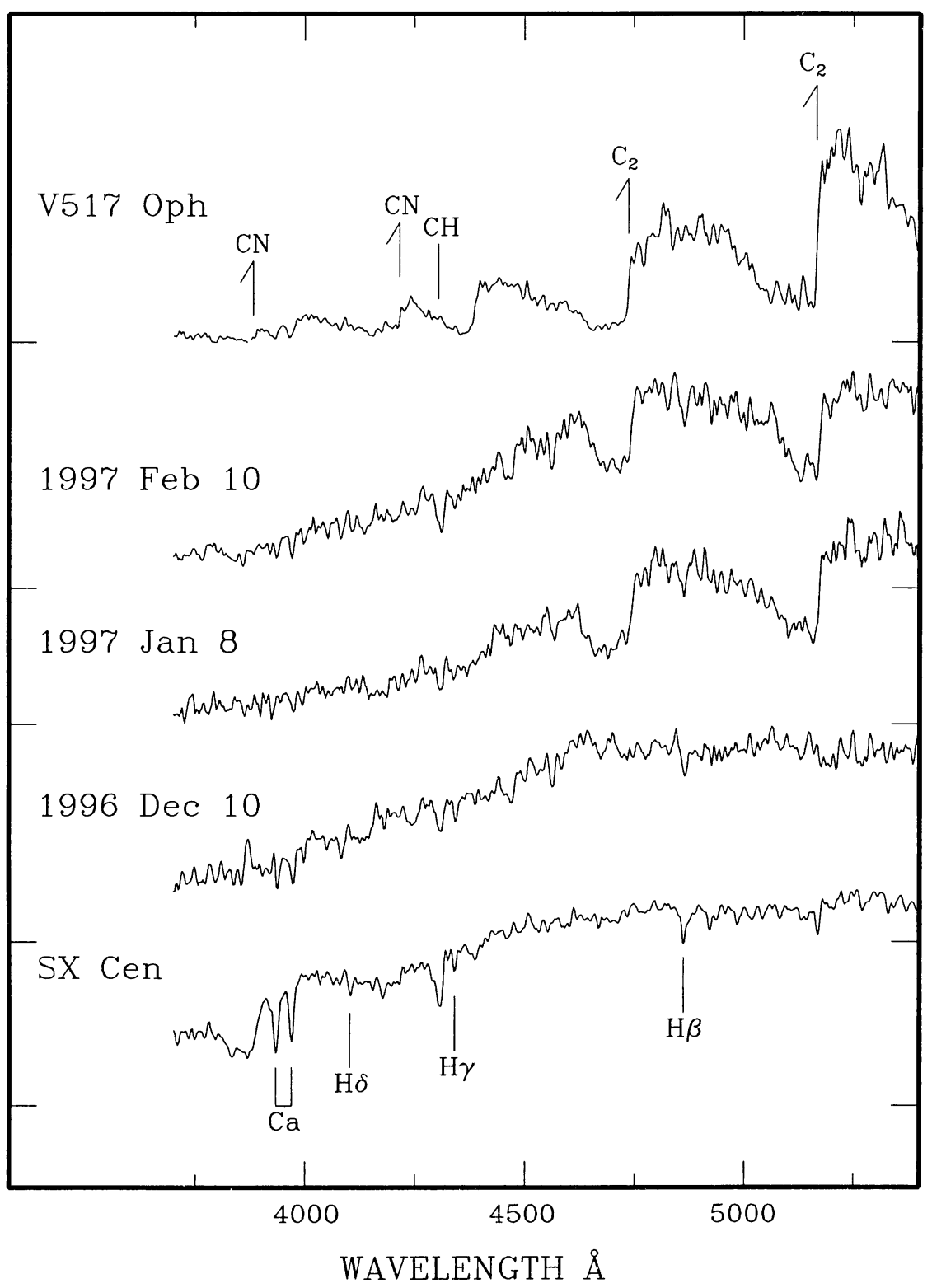

Figure 2. The three low-dispersion spectra of MACHO*04:55:43.267:51:10 (indicated with their dates of observation) are compared with SX Cen, a galactic type 'B' (that is, carbon-rich) RV Tauri star, and V517 Oph, a cool $\mathrm{R}$ Coronae Borealis star that displays relatively strong $\mathrm{C}_{2}$ molecular bands. The ordinate is flux on an arbitrary scale. 
strong $\mathrm{C}_{2}$ bands, but are present and quite pronounced in the spectrum taken near maximum light. The spectra have a low $\mathrm{S} / \mathrm{N}$ in the $4000 \AA$ region and the presence of $\mathrm{C}_{3}$ is uncertain - the strongest bandhead near $4050 \AA$ may be present on the third observation.

The unusual features of the spectrum of MACHO*04:55:43.2-67:51:10 can be matched by stars of another type, the proto-planetary nebulae (Hrivnak \& Kwok 1991; Hrivnak 1995). One of these stars has been found to be variable but the type of variability and the dependence of the spectrum on brightness is unknown. These stars were identified on the strength of large infrared excesses, especially at wavelengths beyond 10 microns, so that there must be a dust shell with a temperature of $150-300 \mathrm{~K}$ at some distance from the star. The JHKL photometry is not expected to show a large excess, being dominated by radiation from the photosphere.

\subsection{Infrared photometry}

The $J H K$ data from SAAO indicates that MACHO*04:55:43.2-67:51:10 has little infrared excess at $\mathrm{K}$ and therefore no substantial warm or hot circumstellar dust. Observations at longer wavelengths would be most useful in detecting the possible presence of cool $(\sim 150-300 \mathrm{~K})$ dust from mass-loss episodes in the past.

\section{Discussion and future work}

MACHO*04:55:43.2-67:51:10 is an RV Tauri variable with a double period of $112.45 \mathrm{~d}$. Three low-resolution $\left(210 \AA \mathrm{mm}^{-1}\right.$ or $7 \AA$ resolution) spectrograms obtained of this star show strong $\mathrm{C}_{2}$ bands with a pronounced phase dependence, their strength being greatest at minimum light. The other spectral features observable with this resolution are all weak and it is impossible to assign a consistent spectral type.

The spectrum of MACHO*04:55:43.2-67:51:10 is similar to those observed in proto-planetary nebulae. It appears that this may be a unique star which combines the main spectral anomaly of the pre-planetary nebulae (PPN) as defined by Hrivnak \& Kwok (1991) and Hrivnak (1995), with RV Tauri-like variability. One might have predicted the existence of such a star, given that, in some cases, the PPN must lie close to the Cepheid instability strip while the RV Tauri variables have been interpreted as post-AGB objects.

The strength of $\mathrm{C}_{2}$ while $\mathrm{CN}$ and $\mathrm{CH}$ are relatively weak is hard to understand. Hrivnak (1995) suggested that $C_{2}$ and $C_{3}$ arose in the extended atmospheres of the pre-planetary stars. The exact relationship of the $\mathrm{C}_{2}$ bandstrength to phase in MACHO*04:55:43.2-67:51:10 is not clearly defined by our observations. However, the large intensity variations of the $C_{2}$ bands suggest strong coupling with the light variations, indicating they probably arise near the stellar photosphere.

The luminosity of MACHO*04:55:43.2-67:51:10 $\left(M_{V}=-3.87\right.$, Alcock et al. 1998) is higher than those typical of the RV Tauri stars present in galactic globular clusters, but there is a rich intermediate-age population in the LMC, typified by the globular clusters, from which such a star might come. Carbon enrichment on the extended AGB of these clusters ultimately results in N-type carbon stars (Bessell, Wood \& Lloyd Evans 1983), so the enhancement of carbon 
is readily understood. The occurrence of a luminous, carbon-rich RV Tauri-like star may be explained by assuming that it is a star of moderately low mass which has moved through the AGB and is now evolving to the blue in the pre-planetary nebula stage. This star may therefore represent a link between these two types of star and strengthens the post-AGB interpretation of RV Tauri stars.

Additional observations are required to clarify the status of this unusual star. One useful datum would be the carbon isotope ratio. A number of RV Tauri stars appear to have enhanced ${ }^{13} \mathrm{C}$ in the cases studied to date (Bujarrabal, Alcolea \& Bachiller 1990; Shenton et al. 1992; Gonzalez, Lambert \& Giridhar 1997a). The various degrees of enhancement of ${ }^{13} \mathrm{C}$ result from different degrees of processing by the $\mathrm{CN}$-cycle and place important constraints on the past evolution of a star. The phase-dependence of the molecular band strengths needs to be delineated. Spectra of improved resolution and $\mathrm{S} / \mathrm{N}$ are required to look for $\mathrm{C}_{3}$ and enhanced s-process elements.

Acknowledgments. We are indebted to Doug Welch for drawing our attention to this star and to the MACHO collaboration for the use of their photometric data. Audrey van der Wielen kindly prepared Fig. 2. We would also like to thank Dave Kilkenny for providing comparison spectra of galactic RCB stars and for a number of very useful discussions.

\section{References}

Alcock C., et al., 1998, AJ 115, 1921

Bessell M. S., Wood P.R., Lloyd Evans T., 1983, MNRAS 202, 59

Bujarrabal V., Alcolea J., Bachiller R., 1990, A\&A 234, 355

Cousins A.W.J., 1981, SAAO Circ. 6, 4

Gingold R.A., 1974, ApJ 193, 177

Gonzalez G., Lambert D.L., 1997, AJ 114, 341

Gonzalez G., Lambert D.L., Giridhar S., 1997a, ApJ 479, 427

Gonzalez G., Lambert D.L., Giridhar S., 1997b, ApJ 481, 452

Hrivnak B.J., 1995, ApJ 438, 341

Hrivnak B.J., Kwok S., 1991, ApJ 371, 631

Jura M., 1986, ApJ 309, 732

Kilkenny D., Lloyd Evans T., Bateson F.M., Jones A.F., Lawson W.A., 1992, The Observatory 112,158

Pollard K.R., et al., 1997, in Variable Stars and the Astrophysical Returns of Microlensing Surveys, R. Ferlet, J.P.Maillard \& B. Raban (eds.), Proc. 12th IAP Astrophysics Colloq., Editions Frontières, Gif-sur-Yvette, p. 219

Pollard K.R., Cottrell P.L., Kilmartin P.M., Gilmore A.C., 1996, MNRAS 279, 949

Pollard K.R., Cottrell P.L., Lawson W.A., Albrow M.D., Tobin W., 1997, MNRAS 286,1

Russell S.C., 1997, A\&A 326, 1069

Schwarzchild M., Harm R., 1970, ApJ 160, 341

Shenton M., et al., 1992, A\&A 262, 138 